\title{
A c-Met inhibitor increases the chemosensitivity of cancer stem cells to the irinotecan in gastric carcinoma
}

\author{
M Yashiro ${ }^{* 1,2}$, T Nishii ${ }^{1}$, T Hasegawa ${ }^{1}$, T Matsuzaki ${ }^{1}$, T Morisaki ${ }^{1}$, T Fukuoka ${ }^{1}$ and K Hirakawa ${ }^{1}$ \\ ${ }^{1}$ Department of Surgical Oncology, Osaka City University Graduate School of Medicine, 1-4-3 Asahi-machi, Abeno-ku, Osaka \\ 545-8585, Japan and ${ }^{2}$ Oncology Institute of Geriatrics and Medical Science, Osaka City University Graduate School of Medicine, \\ 1-4-3 Asahi-machi, Abeno-ku, Osaka 545-8585, Japan
}

Background: Cancer stem cells (CSCs) may be postulated mediators of the chemoresistance. This study aimed to determine an effective signal inhibitor with effects on the proliferation of CSCs in combination with anticancer drugs.

Methods: We used three gastric cancer cell lines and three side population (SP)-enriched CSC cell lines. We examined the combined effects of inhibitors against stemness signals, including c-Met inhibitor SU11274, and five anticancer drugs on the CSC proliferation and mRNA expression of chemoresistance-associated genes.

Results: The IC $\mathrm{C}_{50}$ of irinotecan in SP-enriched CSC was 10.5 times higher than parent OCUM-2M cells, whereas that of oxaliplatin, taxol, gemcitabine, and 5-fluorouracil was 2.0, 2.8, 2.0, and 1.2, respectively. The SP cell lines had higher expression levels of UGT1A1, ABCG2, and ABCB1 than their parent cell lines. There was a synergistic antiproliferative effect with a combination of SU11274 and SN38 in SP cells, but not other inhibitors. The SU11274 significantly decreased the expression of UGT1A1, but not ABCG2 and ABCB1. The SN38 plus SU11274 group more effectively suppressed in vivo tumour growth by OCUM-2M/SP cells than either group alone.

Conclusion: Cancer stem cells have chemoresistance to irinotecan. The c-Met inhibitor may be a promising target molecule for irinotecan-based chemotherapy of gastric cancer.

Gastric cancer remains a major global health threat and most patients with advanced-stage disease require chemotherapy. The development of drug resistance is a major obstacle in the treatment of gastric cancer and only few effective therapies for combating chemoresistance are currently available. It has been demonstrated that a small subset of cancer cells with stem cell properties, referred to as 'cancer stem cells' (CSCs), survive intensive anticancer therapies better than proliferating progenitor cells or differentiated tumour cells (Dean et al, 2005). The CSCs have been reported to be postulated mediators of chemoresistance, and hence it might be important to comprehend the drug-resistance mechanisms of CSCs to develop a promising therapy to combat chemoresistance. Several signals such as c-Met (Li et al, 2011a, b), GSK3 $\beta$ (Reddiconto et al,
2012), and mTOR (Sunayama et al, 2010; Li and Bhatia, 2011) are known to be associated with the stemness of CSCs; therefore, targeting their cellular pathways, which might have important roles in the resistance of CSCs, may provide new treatment modalities for gastric cancer in the advanced stage.

Recent studies have demonstrated that side population (SP) cells are widespread in human cancer cell lines. We also observed that SP cells have properties similar to stem cells (Nishii et al, 2009). The SP cells are determined by the differential potential of cells to efflux the fluorescence dye, Hoechst 33342, via the ATP-binding cassette $(\mathrm{ABC})$ transporter, which is associated with SP chemoresistance properties. The $\mathrm{ABC}$ transporters, including MDR1 (ABCB1), MRP1 (ABCC1), and ABCG2, can confer multidrug 
resistance to cancer cells. The expression of these transporters is correlated with the response to therapy and survival (Gottesman et al, 2002). Prevention of ABC transporter induction in cancer cells might help to avert drug resistance. Camptothecin (CPT-11, SN38, or irinotecan hydrochloride), a DNA topoisomerase I inhibitor, blocks the DNA religation of topoisomerase cleavage complexes (Pommier et al, 1998), and currently it is clinically used as one of the preferred choices in monotherapy or combination therapy for advanced gastric cancer (Bugat, 2003; Arnold et al, 2006; Farhat, 2007). Several phase II studies have evaluated irinotecan monotherapy as first-line chemotherapy for gastric cancer, but the response rate to irinotecan monotherapy is only $\sim 20 \%$ (Kohne et al, 2003).

Recently, small molecules that act as tyrosine kinase inhibitors (TKIs) have begun to emerge as potential therapeutics for use in combination with cytostatic drugs to treat various types of carcinomas. c-Met, the tyrosine kinase receptor for hepatocyte growth factor (HGF), is overexpressed in a variety of tumours where it has an important role during malignant transformation. The c-Met receptor is frequently overexpressed during advanced gastric cancer, and patients with high HGF or c-Met expression have a prognosis worse than those with low expression (Yonemura et al, 1996; Birchmeier et al, 2003; Toiyama et al, 2012). Patients with overexpressed c-Met had a worse prognosis in gastric carcinoma (Drebber et al, 2008; Graziano et al, 2011; Zhao et al, 2011). High expression of HGF and c-Met in gastric cancer tissue is significantly correlated with peritoneal dissemination and poor prognosis (Toiyama et al, 2012). In particular, diffuse type of gastric cancer had a high incidence of Met expression (Kuniyasu et al, 1992; Yonemura et al, 1996). The c-met oncogene amplification might be associated with the development and progression of poorly differentiated gastric cancers (Wang et al, 2004), especially diffuse-type stomach carcinoma (Kuniyasu et al, 1992; Yokozaki et al, 2001). Clinically, conventional chemotherapy resistance found in some types of cancer has been associated with the activation of c-Met expression. In this context, Shah et al (2007) demonstrated that the increased phosphorylation of c-Met was related to gemcitabine resistance in pancreatic cancer.

A combined treatment using a chemotherapeutic agent and a molecular targeting compound might achieve a better response rate than a chemotherapeutic agent alone. However, the effects of a combination of a molecular targeting compound and a chemotherapeutic agent in CSCs of gastric cancer remain to be clarified.

c-Met is known to be a critical signalling molecule during normal stem cell function, but the potential role of c-Met as a single marker of CSCs has not been elucidated.

In the present study, we analysed the effect of c-Met inhibitors on the chemosensitivity of stem-like cancer cells in gastric cancer. We demonstrated that a c-Met inhibitor synergistically increased the antitumour activity of SN38 in CSCs. To determine the mechanisms underlying this observed synergism, we observed that a c-Met inhibitor combined with SN38 also led to a significant increase in UGT1A1 and its subsequent interaction with apoptosisrelated genes, that is, bcl-2 and caspase- 6 .

\section{MATERIALS AND METHODS}

Chemicals and anticancer drugs. Three cell signal inhibitors, c-Met inhibitor SU11274 (Calbiochem, Darmstadt, Germany), GSK3 $\beta$ inhibitor AR-A014418 (Calbiochem), and mTOR inhibitor rapamycin (Sigma, St Louis, MO, USA), were used. Five anticancer drugs, irinotecan (SN38; Yakult, Tokyo, Japan), oxaliplatin (OXA; Yakult), 5-fluorouracil (5FU; Kyowa Hakko, Tokyo, Japan), paclitaxel (PTX; Bristol-Myers, Wallingford, CT), and gemcitabine (GEM; Eli Lilly, Kobe, Japan), were used. All were used according to the protocol providing by the manufacture. The SN38 (Yakult) was dissolved by $1 \mathrm{~mm}$ natrium hydroxydatum at the concentration of $1 \mathrm{M}$, stored at $-20^{\circ} \mathrm{C}$, and diluted to the desired concentration by medium at the $\mathrm{pH}$ from 7.0 to 7.4 .

Cell culture and cell lines. The human gastric cancer cell lines OCUM-2M (Yashiro et al, 1994), OCUM-2D (Yashiro et al, 1995), and OCUM-2MD3 (Yashiro et al, 1996b), which were derived from diffuse types of gastric carcinoma, were used in this study. These cells were cultured in medium consisted of Dulbecco's modified Eagle's medium (DMEM; Nikken Bio., Kyoto, Japan) with the addition of $10 \%$ fetal bovine serum (FBS; Life Technologies, Grand Island, NY, USA), $100 \mathrm{IU} \mathrm{ml}^{-1}$ penicillin (ICN Biomedicals, Costa Mesa, CA, USA), $100 \mu \mathrm{g} \mathrm{ml}^{-1}$ streptomycin (ICN Biomedicals), and $0.5 \mathrm{~mm}$ sodium pyruvate (Cambrex, Walkersville, MD, USA). They were incubated at $37^{\circ} \mathrm{C}$ in an atmosphere containing $5 \% \mathrm{CO}_{2}$. The SP-enriched cell lines, OCUM-2M/SP (Nishii et al, 2009), OCUM-2D/SP (Nishii et al, 2009), and OCUM-2MD3/SP (Nishii et al, 2009), were sorted by FACScan using Hoechst 33342 from OCUM-2M, OCUM-2D (Yashiro et al, 1995), and OCUM-2MD3 (Yashiro et al, 1996b), respectively, as previously reported.

Proliferation assay. The effect of chemotherapeutic drugs with or without the small-synthetic molecules on the proliferation of gastric cancer cells was determined by 3-[4,5-dimethylthiazol-2yl]-2, 5-diphenyltetrazoliumbromide (MTT; Sigma) assay. Cancer cells were seeded into two 96-well plates at a concentration of 10000 cells per well with culture medium exposed to each chemotherapeutic drug at different concentrations and/or SU11274 $(1 \mu \mathrm{M})$, AR-A014418 (35 nM), or rapamycin (20 nM). After incubation for $72 \mathrm{~h}, 20 \mu \mathrm{l}$ of $1 \mathrm{mg} \mathrm{ml}^{-1}$ MTT (Sigma) was added into each well. The formazan product of MTT was measured as absorbance at $570 \mathrm{~nm}$ using a microtitre plate reader (PM2004, Wako, Osaka, Japan). The percentage of cell viability was determined as the ratio of the absorbance of the sample $v s$ the control. Three independent experiments were performed. The potential synergy between signal inhibitors and the anticancer drugs was evaluated using Drewinko's fraction method (Drewinko et al, 1976). The synergistic, additive, and antagonistic interactions were determined when the value was less than the expected value, more than the expected value but less than the drugs' value, and more than the drugs' value, respectively. The expected value of the combined effects $(\%)=$ the effects of the anticancer drug/control $\times$ the effects of cell-signal inhibitor/control $\times 100$ was calculated.

The $\mathrm{IC}_{50}$ of chemotherapeutic drug was determined as each chemotherapeutic drug concentration showing 50\% cell growth inhibition as compared with the control cell growth. Six replicate wells were used for each drug concentration and the testing was carried out independently three times. The potential synergy in combination with small-molecule kinase inhibitors and SN38 was evaluated, using the multiple drug-effect analysis with CalcuSyn software (Version 2.0, Biosoft, Cambridge, UK) including the combination index (CI) method of Chou and Talalay (1984); the $\mathrm{CI}$ indicates synergism $(\mathrm{CI}<0.9)$, additivity $(\mathrm{CI}=0.9-1.1)$, or antagonism $(\mathrm{CI}>1.1)$.

Western blot analysis. Cell lysates were collected after different treatments. After the protein concentration of each sample was adjusted, electrophoresis was carried out using 10\% Tris/Gly gels (Invitrogen, Inc., Gaithersburg, MD, USA). The protein bands obtained were transferred to an Immobilon-P Transfer membrane (Amersham, Aylesbury, UK). The membrane was kept in PBS-T (10 mm PBS and $0.05 \%$ Tween-20) supplemented with 5\% bovine albumin (Sigma) at room temperature for $1 \mathrm{~h}$. Then, the membrane was placed in PBS-T solution containing each primary antibody, p-Met (Y1234/1235; Cell Signaling Technology, Danvers, CO, USA) 
and Met (sc-162; Santa Cruz, Santa Cruz, CA, USA), and allowed to react at room temperature for $2 \mathrm{~h}$. The levels of specific proteins in each lysate were detected by enhanced chemiluminescence using ECL plus (Amersham) followed by autoradiography.

Reverse transcription-PCR. The effects of SN38 or SU11274 on the mRNA expression of the uridine 50-diphosphate-glucuronosyltransferase 1A1 (UGT1A1), ABCG2, ABCB1, ABCC1, ABCC2, p21, p53, Capasase-6, and bcl2 in cancer cells were examined as follows. The cells were plated in six-well microtitre plates at a density of $2 \times 10^{5}$ per well with SN38 at $\mathrm{IC}_{50}$ and/or SU11274, and each plate was incubated for $24 \mathrm{~h}$. After incubation, total cellular RNA was extracted from gastric cancer cells with Trizol (Invitrogen) according to the manufacturer's protocol. The total cellular RNA was extracted using Trizol reagent (Invitrogen) according to the manufacturer's protocol. After the genomic DNA was removed by DNAse, cDNA was prepared from $2 \mu \mathrm{g}$ of RNA with Maloney mouse leukaemia virus reverse transcriptase (Invitrogen) using random primers (Invitrogen). Quantitative real-time RT-PCR was done on the ABI Prism 7000 (Applied Biosystems, Foster City, CA, USA) using the commercially available gene expression assay (Applied Biosystems) for UGT1A1 (Hs01053796), ABCG2 (Hs02511055), ABCB1 (Hs01067802), ABCC1 (Hs00219905), ABCC2 (Hs00166123), p21 (Hs01121172), p53 (Hs01034249), Capasase-6 (Hs00154250), and bcl2 (Hs00608023). Then, PCR was performed at $95^{\circ} \mathrm{C}$ for $15 \mathrm{~s}$ and $60^{\circ} \mathrm{C}$ for $60 \mathrm{~s}$ for 40 cycles. As internal standard to normalise mRNA levels for differences in sample concentration and loading, amplification of glyceraldehyde3-phosphate dehydrogenase $(G A P D H)$ was used. The threshold cycle $\left(C_{\mathrm{t}}\right)$ values were used for calculation of the relative expression ratios between control and treated cells using the formula described by Pfaffl (Pfaffl, 2001). All quantitative PCR reactions were done in triplicate.

Cell cycle test. The cell cycle phase distribution was evaluated using flow cytometry. Cancer cells $\left(2 \times 10^{4}\right.$ cells $)$ were seeded into a $100-\mathrm{mm}$ dish with SN38 or SU11274 $(1 \mu \mathrm{M})$ for the cell cycle test. The concentration of SN38 was $20 \mathrm{nM}$ in OCUM-2M/SP cells and $2 \mathrm{~nm}$ in OCUM-2M cells. After incubation for $72 \mathrm{~h}$, the cells were harvested and managed according to the instructions of the Cycle Test Plus DNA reagent kit protocol (Becton Dickinson, Mountain View, CA, USA), then incubated with ribonuclease A for $10 \mathrm{~min}$ at room temperature, and with propidium iodide for $30 \mathrm{~min}$ in the dark on ice. The sub-G0/G1-, S-, and G2/M-phase fractions of $2 \times 10^{4}$ cells were determined by flow cytometry using a FACScaliber (Becton Dickinson). The results were analysed using the Modofit software program (Beckton Dickinson).

Animal models. The BALB/c nude mice (Clea Japan, Shizuoka, Japan) were used. All experiments with nude mice were performed in accordance with the animal experiments guidelines approved by Osaka City University Ethical Committee. Xenografts were established by injecting $1 \times 10^{7}$ OCUM-2M/SP cells into the flanks of mice at 4 weeks of age. Mean tumour size was observed to be $120 \mathrm{~mm}^{2}$ at 8 days after inoculation. Accordingly, $15 \mathrm{mg} \mathrm{kg}^{-1}$ per day of SN38 and/or $6 \mathrm{mg} \mathrm{kg}^{-1}$ per day of SU11274 was administered for 3 days per week for 2 weeks, except in control. The SN38 and SU11274 were intraperitonealy injected. Tumour volume $(V)$ was determined at each time point by measuring length $(l)$ and width $(w)$, and then calculating the volume $\left(V=l w^{2} / 2\right)$. Medication-defined groups were Vehicle (control group; $n=7)$, SU11274 (6 $\mathrm{mg} \mathrm{kg}^{-1}$ per day; $\left.n=7\right)$, SN38 (15 mg $\mathrm{kg}^{-1}$ per day; $\left.n=6\right)$, and SU11274 combined with SN38 $(n=8)$.

Immunohistochemical techniques. Immunohistochemical determination of Ki67 was examined using xenografted tumours among the four groups. Briefly, paraffin-embedded sections were cut at $5 \mu \mathrm{m}$. The tissue sections were incubated with anti-Ki67 antibody (Zymed, South San Francisco, CA, USA), and were treated with a secondary antibody. The percentage of positive tumour cells was then calculated as Ki67 labelling index (LI) after counting tumour cells in five random fields $(\times 400)$ of each section. Apoptotic cells were determined by DNA using an in situ apoptosis detection kit (Takara, Shiga, Japan). The enzyme, terminal deoxynucleotidyl transferase (TdT), was used to incorporate dioxigenin-conjugated dUTP to the ends of DNA fragments. The signal of TdT-mediated dUTP nick end labelling (TUNEL) was then detected by antidigoxigenin antibody conjugated with peroxidase. The total number of TUNEL-positive cells in five random fields $(\times 400)$ of each section was counted as apoptotic index.

Statistical analysis. Comparisons among the data sets were made by Student's $t$-test. Differences were considered to be statistically significant when the $P$-value was $\leqslant 0.05$.

\section{RESULTS}

The $\mathrm{IC}_{50}$ value of anticancer drugs in SP cells and the parent gastric cancer cell line, OCUM-2M. Figure 1 shows the antiproliferative effects of five anticancer drugs, SN38, PTX, 5FU, OXA, and GEM, in their parent OCUM-2M cells and SP cells, OCUM-2M/SP. The $\mathrm{IC}_{50}$ value (the drug concentration required for $50 \%$ growth reduction in the survival curve) of SN38, PTX, 5FU, OXA, and GEM in OCUM-2M/SP cells was $29.5 \mathrm{nM}, 4.8 \mathrm{nM}$, $2.3 \mu \mathrm{M}, 3.9 \mu \mathrm{M}$, and $94.3 \mathrm{nM}$, respectively, whereas that in their parent OCUM-2M cells was $2.8 \mathrm{nM}, 2.4 \mathrm{~nm}, 2.0 \mu \mathrm{M}, 1.4 \mu \mathrm{M}$, and $47.4 \mathrm{nM}$, respectively. The resistance index (RI) was determined as the ratio of the $\mathrm{IC}_{50}$ of the OCUM-2M/SP cell line $v s$ the $\mathrm{IC}_{50}$ of the parent OCUM-2M. The RI of SN38, PTX, OXA, and GEM in OCUM-2M/SP cells was 10.5, 2.0, 2.8, and 2.0 times higher than their parent OCUM-2M cells, respectively, whereas the $\mathrm{IC}_{50}$ of $5 \mathrm{FU}$ (1.2 times) did not differ between the two cell lines. The RI of SN38 was the highest of the five anticancer drugs in OCUM-2M/SP and OCUM-2M.

Combined effect of signal inhibitors and anticancer drugs. Next, we investigated the antiproliferative effect of signal inhibitors, including the c-Met inhibitor SU11274, GSK3 $\beta$ inhibitor AR-A014418, and mTOR inhibitor rapamycin, on gastric cancer cells when combined with anticancer drugs, such as SN38, PTX, OXA, or GEM (Table 1). A synergistic antiproliferative effect was found only for a combination of the c-Met inhibitor with SN38 in the OCUM-2M and OCUM-2M/SP cells. In contrast, the GSK3 $\beta$ inhibitor had no synergistic effect when combined with any of the anticancer drugs. An additive effect was found for combinations of the c-Met inhibitor, GSK $3 \beta$ inhibitor, or mTOR inhibitor with SN38, PTX, OXA, or GEM, in each cell line. An antagonistic effect was found with other combinations of signal inhibitors and anticancer drugs in either OCUM-2M or OCUM$2 \mathrm{M} / \mathrm{SP}$ cells. We then investigated the effect of a combination of the c-Met inhibitor with SN38 in detail, as described below.

Effect of SU11274 on the $\mathrm{IC}_{50}$ value of parent cells and their SP cells in three gastric cancer cell lines. Figure 2A shows the cell growth inhibition curve of SN38 in the presence or absence of $2 \mu \mathrm{M}$ SU11274 in the parent cells OCUM-2M, OCUM-2D, and OCUM-2MD3, and their SP cells OCUM-2M/SP, OCUM-2D/SP, and OCUM-2MD3/SP. The $\mathrm{IC}_{50}$ value of SN38 in OCUM-2M/SP (29.5 nM), OCUM-2D/SP (19.0 nM), and OCUM-2MD3/SP $(11.8 \mathrm{nM})$ was higher than that in the parent cells OCUM-2M (2.8 nM), OCUM-2D (7.7 nM), and OCUM-2MD3 (5.4 nM). In the presence of the c-Met inhibitor SU11274 $(2 \mu \mathrm{M})$ the $\mathrm{IC}_{50}$ value of SN38 was reduced from 29.5 to $3.8 \mathrm{~nm}$ in OCUM-2M/SP cells, from 19.0 to $2.6 \mathrm{nM}$ in OCUM-2D/SP cells, and from 11.8 to $2.5 \mathrm{nM}$ in OCUM-2MD3/SP cells. The $\mathrm{IC}_{50}$ value of SN38 in all three SP 

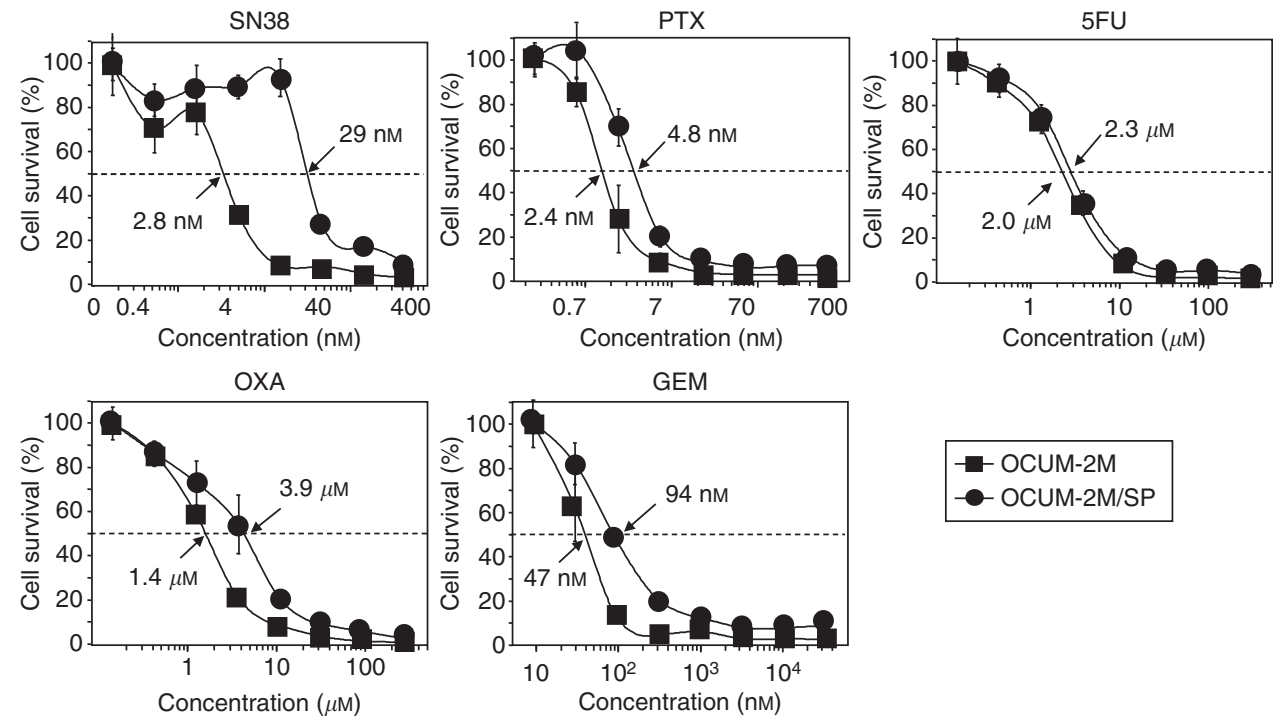

Figure 1. The $\mathrm{IC}_{50}$ of anticancer drugs in SP cells (OCUM-2M/SP) and their parent gastric cancer cells (OCUM-2M). A human gastric cancer cell line, OCUM-2M ( $\mathbf{\square})$, and side population subline, OCUM-2M/SP $(\bullet)$, were exposed to each chemotherapeutic drug at different concentrations. The IC $\mathrm{C}_{50}$ of SN38, PTX, OXA, and GEM in OCUM-2M/SP cells was higher than in their parent OCUM-2M cells, but not IC 50 of 5FU.

\begin{tabular}{|c|c|c|c|c|c|c|c|c|}
\hline \multirow[b]{2}{*}{ Signal inhibitor } & \multicolumn{4}{|c|}{ OCUM-2M } & \multicolumn{4}{|c|}{ OCUM-2M/SP } \\
\hline & SN38 & PTX & OXA & GEM & SN38 & PTX & OXA & GEM \\
\hline SU11274 (c-Met inhibitor) & Synergistic & - & - & - & Synergistic & - & - & - \\
\hline AR-A014418 (GSK3 $\beta$ inhibitor) & Additive & - & Additive & - & - & - & - & - \\
\hline Rapamycin (mTOR inhibitor) & - & - & Additive & - & Additive & Additive & - & Additive \\
\hline
\end{tabular}

cells was reduced by one-fourth or less on co-exposure to SN38 and SU11274 $(2 \mu \mathrm{M})$ as compared with SN38 alone.

Synergistic effects of SU11274 on the antiproliferative efficiency of SN38. Figure 2B shows the effects of SU11274 on the antiproliferative efficiency of SN38, which were analysed using the CalcuSyn software program. The effects of these combinations of a c-Met inhibitor with $\mathrm{SN} 38$ were synergistic $(\mathrm{CI}<0.9)$ in OCUM-2M/SP, OCUM-2D/SP, and OCUM-2MD3/SP. In contrast, antagonism $(\mathrm{CI}>1)$ was detected at some points in the parent cells OCUM-2M, OCUM-2D, and OCUM-2MD3.

Effects of HGF or SU11274 on the c-Met phosphorylation of gastric cancer cells. The inhibitory effect of SU11274 on c-Met phosphorylation was determined by immunoblotting. Hepatocyte growth factor increased the c-Met phosphorylation of gastric cancer cells. Phosphorylation of c-Met was decreased by $\geqslant 1 \mu \mathrm{M}$ of SU11274 in OCUM-2M/SP, OCUM-2D/SP, and OCUM-2MD3/SP cells. In contrast, a difference in phospho-c-Met expression was evident with any SU11274 concentration compared with the total c-Met expression in the parent cells OCUM-2M, OCUM-2D, and OCUM-2MD3 (Figure 3).

Effects of SU11274 on mRNA expression in gastric cancer cells. The expression levels of UGT1A1, ABCG2, ABCB1, and caspase- 6 were significantly increased in all three SP cell lines compared with their parent cell lines, whereas the expression level of $b c l-2$ was significantly decreased. SU11274 significantly decreased the expression of UGT1A1 and caspase-6, and decreased that of $b c l-2$ compared with the control in all three SP cell lines, but not the parent cell lines. The combination of SU11274 and SN38 significantly increased the levels of apoptosis-related gene $p 21$ and significantly decreased the levels of UGT1A1 and ABCG2 compared with SN38 alone (Figure 4). In contrast, we observed no significant difference in the mRNA expression of $A B C C 1$, $A B C C 2$, or $p 53$ in the presence of SU11274 and/or SN38 in all cell lines (data not shown).

Cell cycle assay of SP and parent cells. The percentage of S-phase OCUM-2M/SP cells (40\%) was lower than that of OCUM-2M cells (51\%). The SU11274 increased the proportion of OCUM-2M/SP cells (47\%). A combination of SN38 and SU11274 increased the proportion of OCUM-2M/SP cells (98\%; Figure 5).

Effect of SU11274 and/or SN38 on OCUM-2M/SP tumour development in vivo. The mean volumes of the subcutaneous tumour of the control, SU11274, SN38, and SU11274 plus SN38 groups were $351,344,185$, and $106 \mathrm{~mm}^{3}$ at day 12 after administration, respectively. The size of tumours in mice receiving the combination of SN38 with the SU11274 was significantly $(P=0.047)$ smaller than in those receiving SN38 alone. The significant difference of tumour size between SN38 alone and SN38 with SU11274 was found between day 7 and day 12 after administration. The synergistic effect was determined when the 

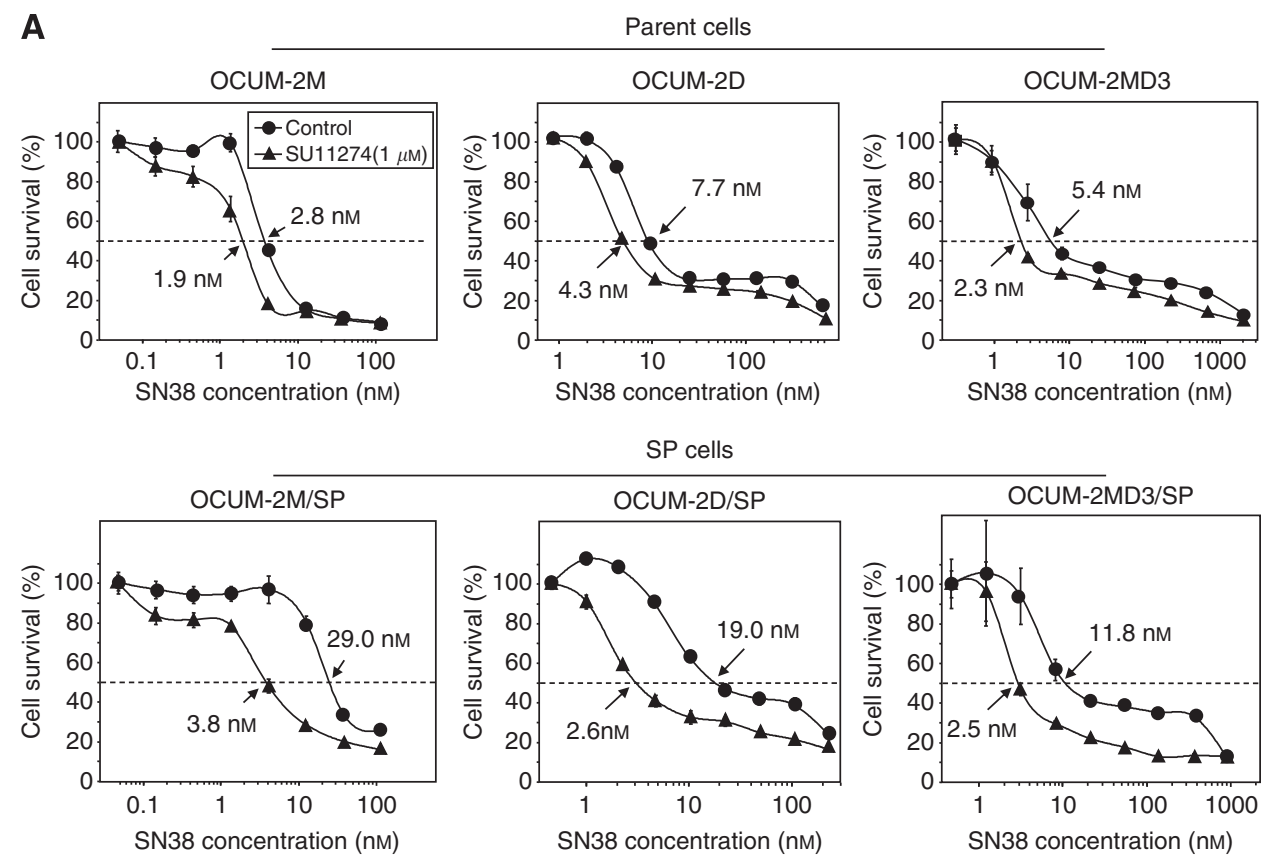

B
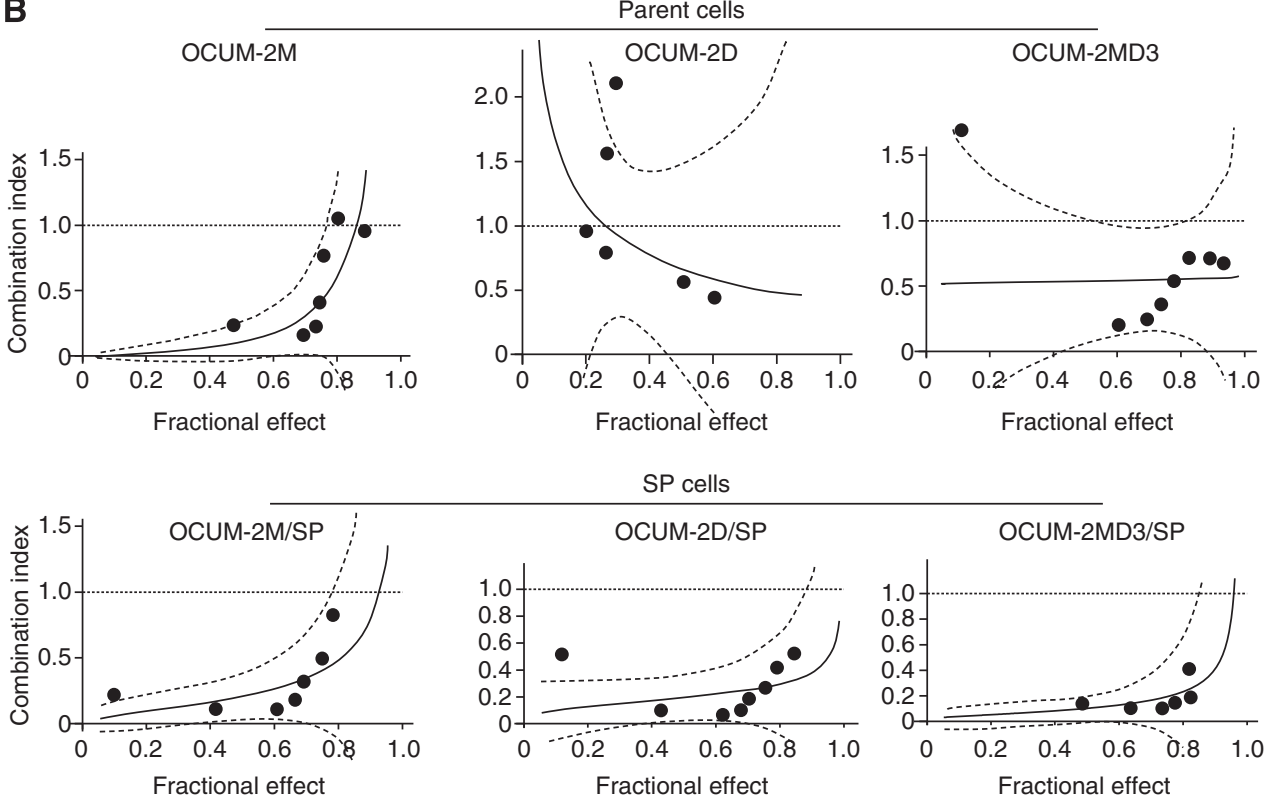

Figure 2. Combination effects of SU1127 with SN38 in parent cell lines and their SP cell lines. (A) The IC 50 value of parent cells and their SP cells. The $\mathrm{IC}_{50}$ values of SN38 in SP cells were higher than those in the parent cells. A combination of the c-Met inhibitor SU11274 and SN38 ( $\mathbf{A}$ ) suppressed the proliferation of all parent cell lines and the SP cell lines compared with SN38 alone ( ). The IC 50 value of SN38 in all three SP cells was clearly decreased by co-exposure to SN38 and SU11274 compared with those of the parent cells. (B) Synergistic effects of SU11274 on the antiproliferative efficiency of SN38. The Cl plots were generated using CalcuSyn software. The effects of the combination of a c-Met inhibitor and SN38 were synergistic $(\mathrm{Cl}<0.9)$ in the SP cells OCUM-2M/SP, OCUM-2D/SP, and OCUM-2MD3/SP. In contrast, antagonism (Cl > 1) occurred at some points in the parent cells of OCUM-2M, OCUM-2D, and OCUM-2MD3. Dotted line, $\mathrm{Cl}=0$.

value by the combination of SU11274 with SN38 was less than the expected value, as previously reported (Marth et al, 1986). The expected value $\left(\mathrm{mm}^{3}\right)$ of the combined effects $=$ the effects of SN38 $\left(\mathrm{mm}^{3}\right) \times$ the effects of SU11274 $\left(\mathrm{mm}^{3}\right)$ /vehicle control $\left(\mathrm{mm}^{3}\right)$. The OCUM-2M/SP tumour size by the combination of SU11274 and SN-38 was lower than the expected value (dotted line). In contrast, no significant difference of tumour size between control and SU11274 was found (Figure 6A).

Effect of SU11274 on the proliferative activity and tumour apoptosis in vivo. Figure 6B shows representative picture of Ki67 staining and apoptotic cells that were TUNEL positive. The proliferative activity of OCUM-2M/SP tumours evaluated by Ki67 LI was $64 \pm 5,60 \pm 4,51 \pm 5$ and $42 \pm 5$ in the control, SU11274-treated, SN38-treated, and SU11274 plus SN38-treated mice, respectively. The Ki67 LI of tumours in mice receiving SU11274 and SN38 was significantly $(P<0.01)$ lower than that in the controls. The TUNEL staining revealed that the numbers of apoptotic cells were increased by SU11274 or SN38 treatment. The apoptotic indices of tumours were $3.5 \pm 1.0,4.1 \pm 1.3,3.9 \pm 1.6$, and $7.5 \pm 1.7$ in the control, SU11274-treated, SN38-treated, and SU11274 plus SN38-treated mice, respectively. A combination of 


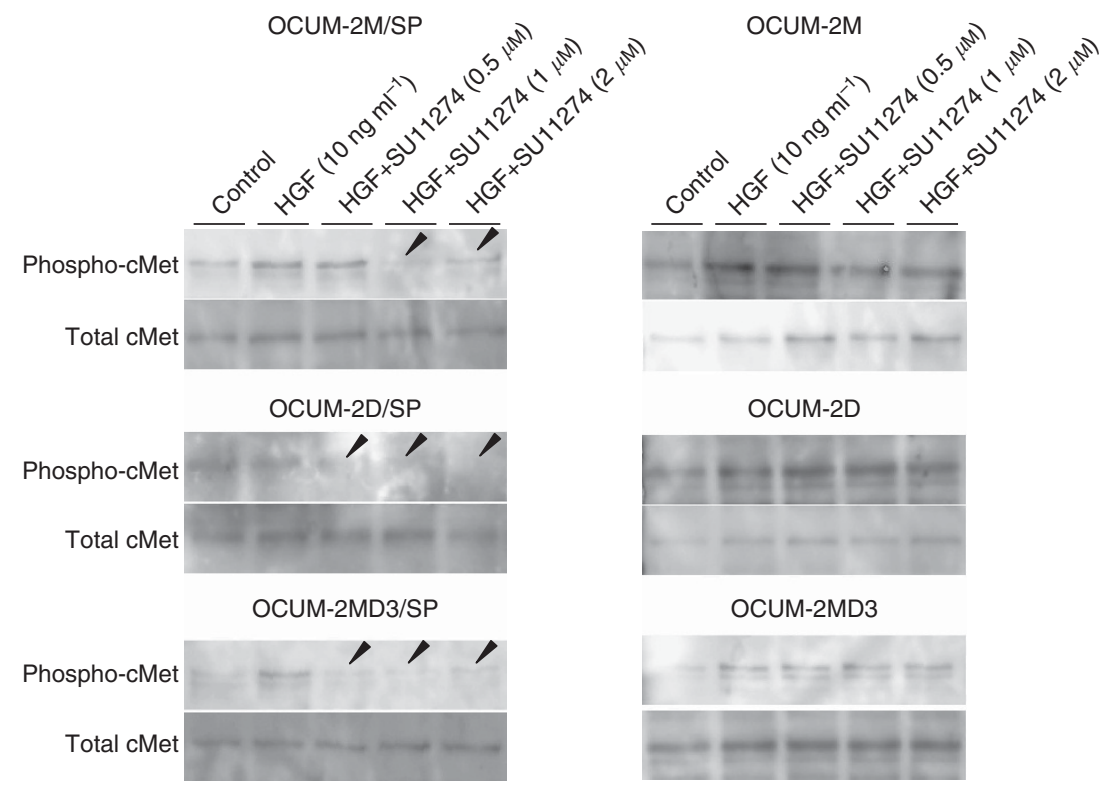

Figure 3. Effects of SU11274 on c-Met phosphorylation. The ability of SU11274 to inhibit c-Met phosphorylation was evaluated in gastric cancer cell lines. SU11274 (>1 $\mu \mathrm{M})$ inhibited the phosphorylation of c-Met in three SP cell lines, but no inhibition was evident in the parent cell lines.

SU11274 plus SN38 significantly induced a greater degree of apoptosis in the primary tumours in comparison with that seen in the control and SN38-treated mice (Figure 6C).

\section{DISCUSSION}

Anticancer therapies are effective against proliferating progenitor cells or differentiated tumour cells, but CSCs can survive chemotherapy and produce progenitor cells or differentiated tumour cells (Dean et al, 2005; Hong et al, 2009). Therefore, the development of a therapy against CSC is important for increasing the chemosensitivity of carcinomas. It would be useful to use CSC cell lines, but there are no reports of the establishment of gastric CSCs. As we previously reported that SP cell lines, identified and isolated by the ability to efflux Hoechst 33342 dye, possessed CSClike properties such as higher tumour formation and metastasis (Nishii et al, 2009), we used these SP cell lines, OCUM-2M/SP, OCUM-2D/SP, and OCUM-2MD3/SP, in this study. Although all of SP cells are not cancer stem cells, SP cells contain high population of cancer stem cells. The $\mathrm{IC}_{50}$ of SP cells with SN38, OXA, PTX, and GEM was 10.5 times, 2.0 times, 2.8 times, and 2.0 times higher than those of their parent cells, respectively. In contrast, the $\mathrm{IC}_{50}$ with $5 \mathrm{FU}$ did not differ between the two cell lines. These findings suggest that CSC-like SP cells have chemoresistance to SN38, OXA, PTX, and GEM. The RI was highest in SN38 among the five anticancer drugs, which suggests that the clinical efficacy of CPT11-based chemotherapy may be dependent on the therapeutic efficacy of SN38 against CSCs.

Next, we examined the effect of three signal inhibitors, c-Met inhibitor SU11274, GSK3 $\beta$ inhibitor AR-A014418, and mTOR inhibitor rapamycin, in combination with anticancer drugs, except 5FU. A synergistic effect on proliferation occurred only with the combination of the c-Met inhibitor and SN38. Therefore, we investigated the effect of a combination of the c-Met inhibitor with SN38 in detail. The $\mathrm{IC}_{50}$ of SN38 in combination with SU11274 was decreased in all three SP cell lines compared with the three parent cell lines. In addition, CalcuSyn analysis indicated that the combination of a c-Met inhibitor and SN38 had synergistic effects in SP cells but not in their parent cells. Taken together, the in vivo tumour by OCUM-2M/SP cells was significantly decreased by SN38 plus c-Met inhibitor compared with the size resulting from SN38 alone. The OCUM-2M/SP tumour size by the combination of SU11274 and SN-38 was lower than the expected value, evaluating that the combination of SU11274 and SN-38 might show a synergistic effect for cancer stem cells. These findings suggest that c-Met inhibitors might enhance the sensitivity to SN38 in SP cells; therefore, c-Met inhibitors may be promising tools for the targeted therapy of human gastric cancer. The role of c-Met in CSC has been determined unequivocally in numerous tumours during the past decade. Additional treatment using c-Met inhibitors could be a novel strategy to overcome chemoresistance. Next, we analysed the mechanism of the synergistic effect using a combination of SN38 and SU11274 in SP cells.

The mRNA expression of ABC transporters, ABCG2 and $A B C B 1$, was significantly higher in SP cell lines than in their parent cell lines. The ABC transporters are widely associated with chemoresistance to several anticancer drugs, including SN38, OXA, PTX, and GEM (Gottesman et al, 2002). Cancer stem cells with a high level of $\mathrm{ABC}$ transporter might have a high capacity for anticancer drug efflux, as previously reported (Kondo et al, 2004; Haraguchi et al, 2006). One of the mechanisms underlying the widespread chemoresistance of SP cells might be the high expression of $\mathrm{ABCG} 2$ and $\mathrm{ABCB} 1$. The mRNA expression of UGT1A1 was significantly higher in SP cell lines than in their parent cell lines. The SN38 is mainly metabolised by UGT1A1 to an inactive metabolite SN38 glucuronide (SN38G) (Innocenti et al, 2005). Therefore, altered expression of an efflux transporter and metabolite enzyme might be involved in the decreased level of SN38 in cells. The high expression of UGT1A1 in SP cells might explain why the RI of SN38 was the highest of the five anticancer drugs. Cancer stem cells possessed resistance to CPT-11 because of decreasing intracellular SN38 levels that resulted from not only an increase in $\mathrm{ABC}$ transporters but also an increase in metabolic enzymes.

It has been reported that SU11274 targets the ATP-binding site of the HGF receptor c-Met and it blocks the kinase activity of Met (Sattler et al, 2003). Our study also revealed that $>1 \mu \mathrm{M} \mathrm{SU11274}$ inhibited the phosphorylation of c-Met in SP cancer cells. The effect of SU11274 was analysed at the concentration of $1 \mu \mathrm{M}$. 

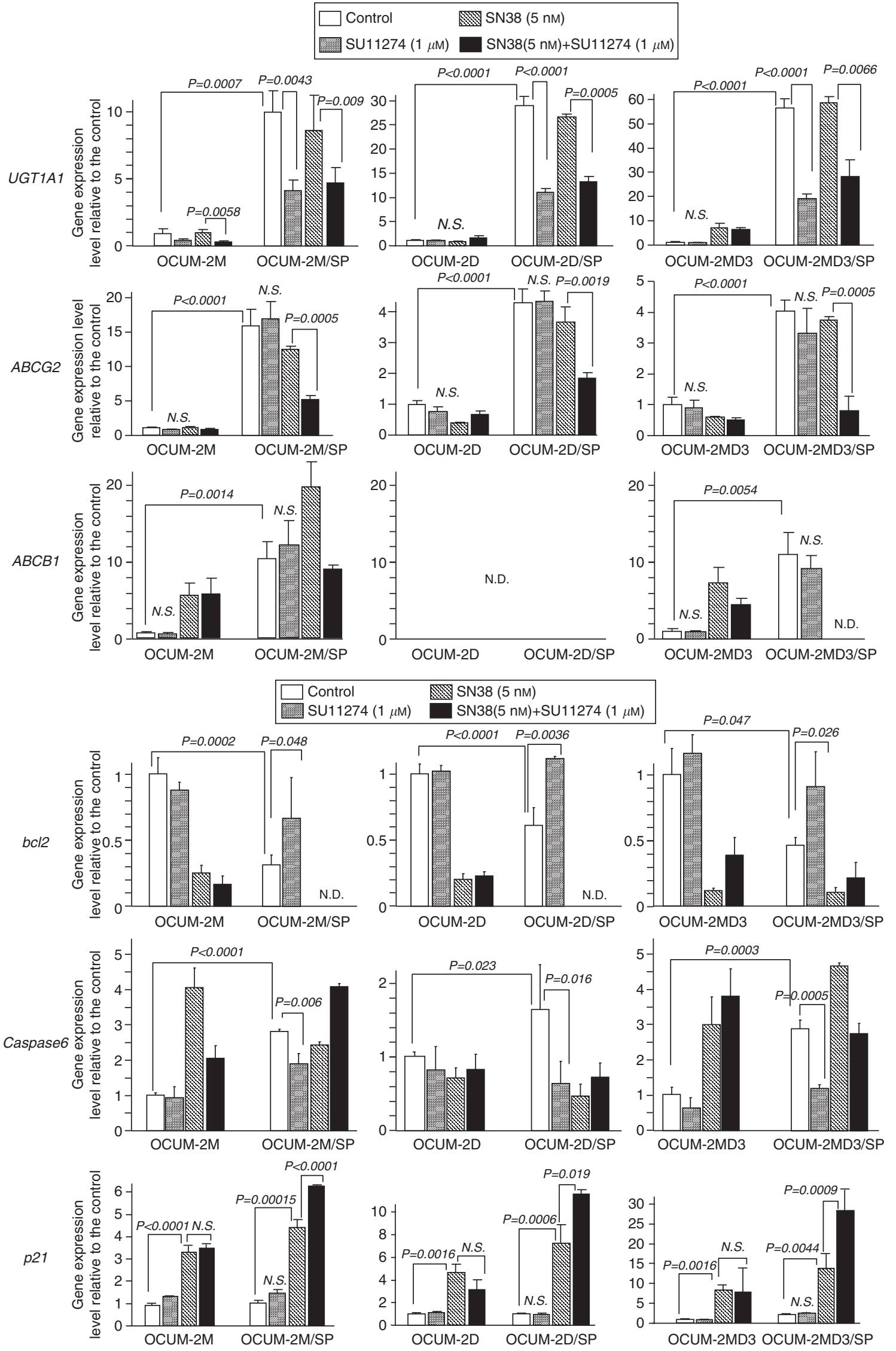

Figure 4. Effects of SU11274 on the mRNA expression of SN38 metabolism-related genes. Three SP cell lines, OCUM-2M/SP, OCUM-2D/SP, and OCUM-2MD3/SP, had expression levels of UGT1A1, ABCG2, ABCB1, and caspase-6 higher than their parent cell lines. In contrast, the three SP cell lines had lower expression levels of bcl-2 than their parent cell lines. The SU11274 significantly decreased the expression of UGT1A1 and caspase-6, and decreased that of bcl-2 compared with the control in all three SP cell lines, but not in the parent cell lines. Both SN38 and SU11274 significantly decreased the UGT1A1 and ABCG2 expression levels and increased the p21 level compared with SN38 alone. ND= not determined; NS=not significant. 


\section{OCUM-2M}
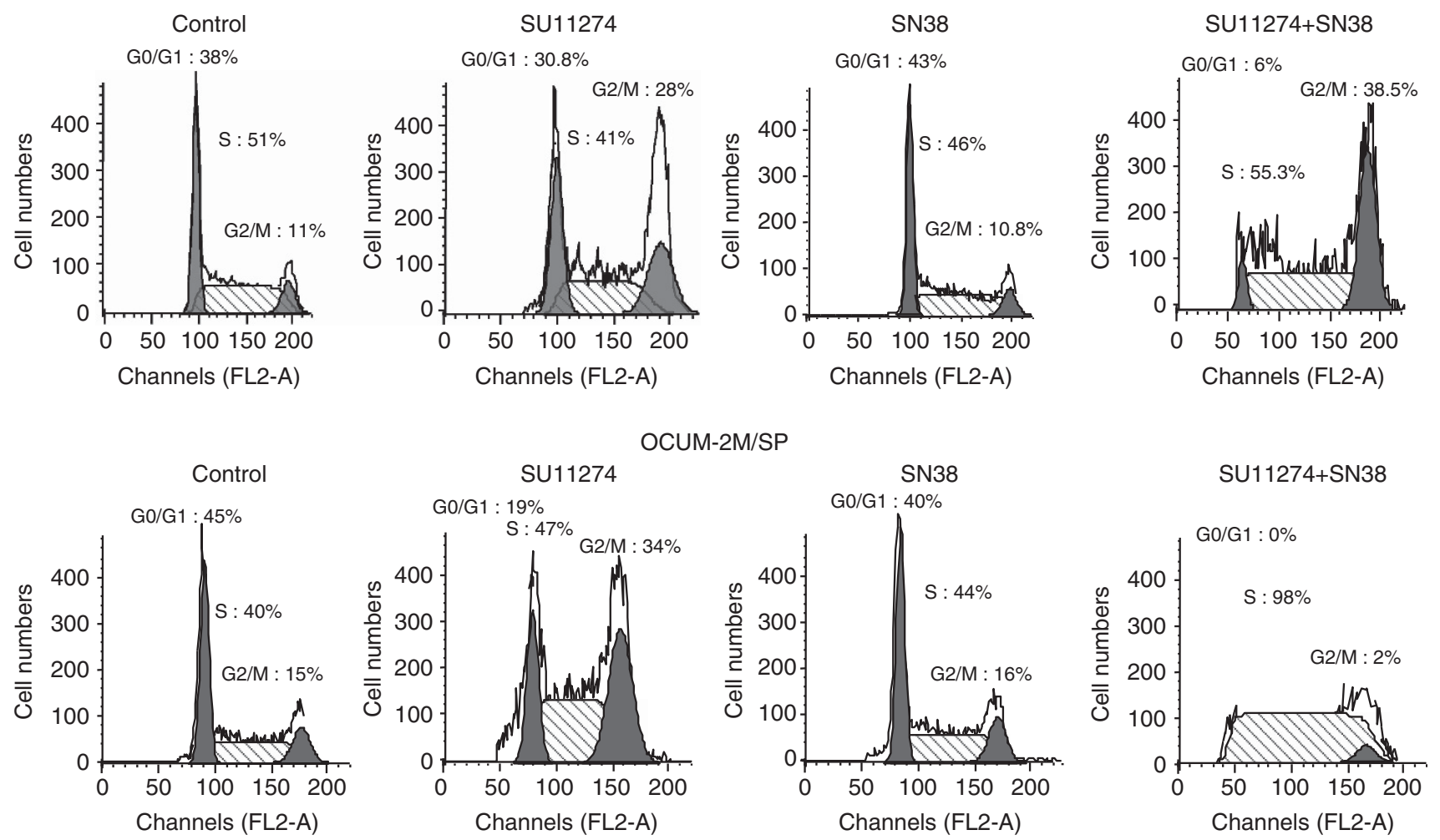

Figure 5. Effects of SU11274 inhibitors on the cell cycle. The proportion of S-phase OCUM-2M/SP cells was low compared with that of OCUM-2M cells. The proportion of S-phase OCUM-2M/SP cells was significantly increased after the addition of SU11274 compared with the control. The combination of SN38 and SU11274 increased the proportion of S-phase OCUM-2M/SP cells.

The SU11274 significantly decreased the expression of UGT1A1 as compared with the control in all three SP cell lines, but not that of $A B C G 2$ and $A B C B 1$. Downregulation of UGT1A1 expression by SU11274 might explain why SU11274 synergistically decreased the SN38-resistant activity of SP cells. The lack of any significant effect of SU11274 on ABCG2 and ABCB1 might explain why the chemosensitivity of SP cells to OXA, 5FU, PTX, and GEM was not improved by pretreatment with SU11274. Combination therapy with a c-Met inhibitor might provide a better response with CPT-11 in gastric carcinomas as compared with therapy using CPT-11 alone. However, the combination of a c-Met inhibitor with other anticancer drugs, OXA, PTX, and GEM, was antagonistic. Thus, it is clinically important to select the appropriate anticancer drug when a c-Met inhibitor is used in combination.

For CSCs, it is reported that their cell cycle proceeds slowly or they remain in the G0 phase (Guzman et al, 2002; Jedema et al, 2004; Fillmore and Kuperwasser, 2007). In this study, the proportion of G0-phase OCUM-2M/SP cells was higher than their parent OCUM-2M cells. Anticancer drugs act when cancer cells are in mitosis or DNA synthesis. The SU11274 increased the proportion of S-phase SP cells. Irinotecan is an S phase-specific, semisynthetic derivative of camptothecin that interferes with DNA replication and cell division by inhibiting topoisomerase I. Its mechanism of action differs from fluoropyrimidines, platinum, and taxanes. The expression levels of a proliferation marker Ki67 was upregulated by the combination of SU11274 with SN38. Alterations in the S phase of SP cells by SU11274 might partly explain the mechanisms responsible for the synergistic antiproliferation effect of SU11274 with SN38. These findings provide a rationale for the antitumour activity and the cell cycle effects of a combination of SU11274 and SN38. The upregulation of the S phase in SN38resistant cell lines by c-Met inhibitors might be responsible for the increased apoptosis during combination therapy with SN38. The apoptosis rates induced by the combination of SU11274 with SN38 were more increased than those by SN38 alone. The mRNA expression of caspase- 6 was increased whereas that of $b c l-2$ was decreased in OCUM-2M/SP cells as compared with OCUM-2M cells. The SU11274 affected the expression level of caspase- 6 and $b c l-2$ in all SP cells, but not of their parent cells. Caspase- 6 and bcl-2 might be associated with cell cycle alterations caused by the c-Met inhibitor in CSC cells. Caspase- 6 and bcl- 2 are involved with cell apoptosis (Korzeniewska-Dyl, 2008). The bcl-2 signalling is induced by a c-Met inhibitor, which might contribute to the induction of apoptosis in SP cancer cells. The regulation of $b c l-2$ and caspase- 6 by c-Met inhibitors might influence the apoptotic effect of SN38 in irinotecan-refractory CSCs.

The HGF protein detected in tumour tissues originates from neighbouring fibroblasts (Yashiro et al, 1996a; Inoue et al, 1997; Yashiro and Hirakawa, 2010). Stem cells reside in a special microenvironment called niche, and the interaction between CSCs and niche plays a critical role in maintenance of stemness, the selfrenewal, and the pluripotency (Iwasaki and Suda, 2009). We previously reported that interaction between cancer cells and HGF from neighbouring fibroblasts plays an important role in the progression of diffuse-type gastric cancers (Yashiro and Hirakawa, 2010), which might suggest that niche fibroblasts affect CSCs of diffuse-type gastric cancer. c-Met is activated in human gastric carcinoma tissue, especially in diffuse-type gastric cancers, and it may produce significant biological effects that mediate antiapoptotic signals (Inoue et al, 2004; Tahara, 2004). The cell lines used in this study were derived from diffuse-type gastric cancer. The combined effect of a c-Met inhibitor with SN38 might be effective for treating the CSCs in diffuse-type gastric cancers.

In conclusion, the CSCs in gastric cancer cells might be associated with chemoresistance. In gastric cancer, UGT1A1 is involved in the development of drug resistance to irinotecan. Blockage of c-Met signalling might contribute to the antitumour effects in irinotecan-refractory gastric cancer by decreasing the 


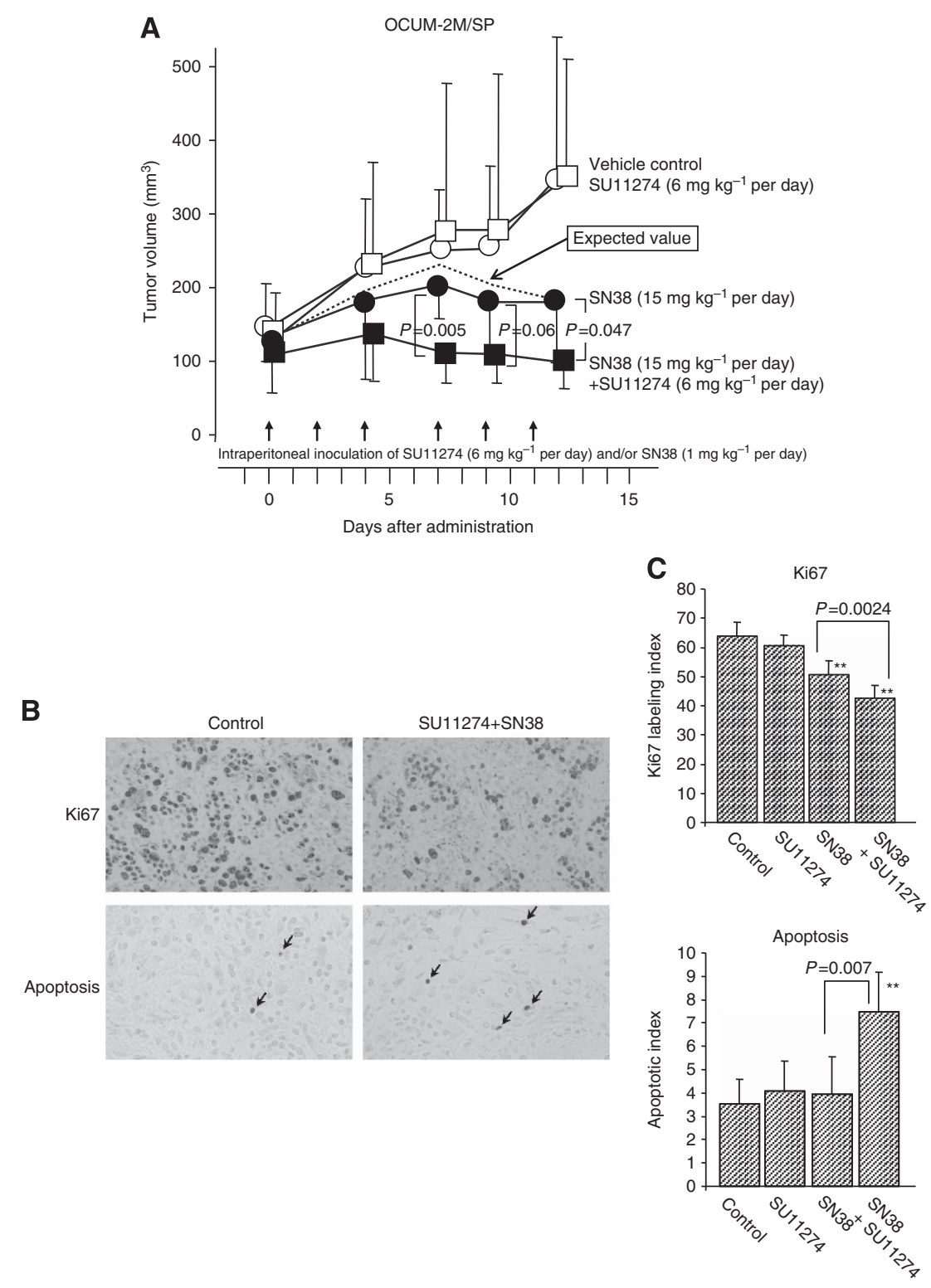

Figure 6. Effect of the combination of SN38 with SU11274 on the proliferation of xenografted tumour in vivo. (A) The OCUM-2M/SP tumour size was significantly decreased by SN38 plus SU11274 ( $\mathbf{\square}$ ) compared with the size resulting from SN38 alone treatment ( $)$. The significant difference of tumour size between SN38 alone and SN38 with the SU11274 was found between day 7 and day 12 after administration. In contrast, no significant difference of tumour size between vehicle control $(O)$ and SU11274 treatment $(\square)$ was found. The OCUM-2M/SP tumour size by the combination of SU11274 and SN-38 was lower than the expected value (dotted line). The expected value of the combined effects = the effects of SN38 $\times$ the effects of SU11274 /vehicle control. (B) The Ki67 immunostaining produced discernible diffuse or granular, brown nuclear staining, more accentuated in the nucleoli, with relatively uniform intensity. The reactivity is more intense in the control compared with SN38 plus SU11274 treatment $(\times 400)$. Apoptotic cells (arrows) show condensation and aggregation of nuclear chromatin into dense masses beneath nuclear membrane $(\times 400)$. (C) Treatment with SN38 alone or SN38 plus SU11274 significantly reduced Ki67 LI in tumour $(P<0.01)$ as compared with the control. The Ki67 LI in mice receiving SN38 plus SU11274 was significantly $(P=0.0024)$ reduced than that in mice receiving SN38 alone. Number of apoptotic (TUNEL-positive) cells in primary gastric tumour was significantly increased following SN38 plus SU11274 treatment as compared with no treatment. Number of apoptotic cells in mice receiving SN38 plus SU11274 treatment was significantly $(P=0.007)$ higher than those in mice receiving SN38 alone. Data are presented as mean \pm s.d. ${ }^{\star \star} P<0.01$ vs control.

UGT1A1 level. A c-Met inhibitor might be a promising molecular targeting compound for combating CSCs with SN38 resistance in human gastric cancers.

\section{ACKNOWLEDGEMENTS}

This study is partially founded by KAKENHI (Grant-in-Aid for Scientific Research, Nos. 20591573, 22390262, and 23390329), by the
National Cancer Center Research and Development Fund (23-A-9), and by Foundation for Promotion of Cancer Research. We thank Masako Shinkawa (Osaka City University Graduate School of Medicine) for technical advice on RT-PCR analysis and western blot.

\section{CONFLICT OF INTEREST}

The authors declare no conflict of interest. 


\section{REFERENCES}

Arnold D, Peinert S, Voigt W, Schmoll HJ (2006) Epidermal growth factor receptor tyrosine kinase inhibitors: present and future role in gastrointestinal cancer treatment: a review. Oncologist 11(6): 602-611.

Birchmeier C, Birchmeier W, Gherardi E, Vande Woude GF (2003) Met, metastasis, motility and more. Nat Rev Mol Cell Biol 4(12): 915-925.

Bugat R (2003) Irinotecan in the treatment of gastric cancer. Ann Oncol 14(Suppl 2): ii37-ii40.

Chou TC, Talalay P (1984) Quantitative analysis of dose-effect relationships: the combined effects of multiple drugs or enzyme inhibitors. Adv Enzyme Regul 22: 27-55.

Dean M, Fojo T, Bates S (2005) Tumour stem cells and drug resistance. Nat Rev Cancer 5(4): 275-284.

Drebber U, Baldus SE, Nolden B, Grass G, Bollschweiler E, Dienes HP, Holscher AH, Monig SP (2008) The overexpression of c-met as a prognostic indicator for gastric carcinoma compared to p53 and p21 nuclear accumulation. Oncol Rep 19(6): 1477-1483.

Drewinko B, Green C, Loo TL (1976) Combination chemotherapy in vitro with cis-dichlorodiammineplatinum(II). Cancer Treat Rep 60(11): 1619-1625.

Farhat FS (2007) A general review of the role of irinotecan (CPT11) in the treatment of gastric cancer. Med Oncol 24(2): 137-146.

Fillmore C, Kuperwasser C (2007) Human breast cancer stem cell markers CD44 and CD24: enriching for cells with functional properties in mice or in man? Breast Cancer Res 9(3): 303.

Gottesman MM, Fojo T, Bates SE (2002) Multidrug resistance in cancer: role of ATP-dependent transporters. Nat Rev Cancer 2(1): 48-58.

Graziano F, Galluccio N, Lorenzini P, Ruzzo A, Canestrari E, D’Emidio S, Catalano V, Sisti V, Ligorio C, Andreoni F, Rulli E, Di Oto E, Fiorentini G, Zingaretti C, De Nictolis M, Cappuzzo F, Magnani M (2011) Genetic activation of the MET pathway and prognosis of patients with high-risk, radically resected gastric cancer. J Clin Oncol 29(36): 4789-4795.

Guzman ML, Swiderski CF, Howard DS, Grimes BA, Rossi RM, Szilvassy SJ, Jordan CT (2002) Preferential induction of apoptosis for primary human leukemic stem cells. Proc Natl Acad Sci USA 99(25): 16220-16225.

Haraguchi N, Inoue H, Tanaka F, Mimori K, Utsunomiya T, Sasaki A, Mori M (2006) Cancer stem cells in human gastrointestinal cancers. Hum Cell 19(1): 24-29.

Hong SP, Wen J, Bang S, Park S, Song SY (2009) CD44-positive cells are responsible for gemcitabine resistance in pancreatic cancer cells. Int $J$ Cancer 125(10): 2323-2331.

Innocenti F, Liu W, Chen P, Desai AA, Das S, Ratain MJ (2005) Haplotypes of variants in the UDP-glucuronosyltransferase1A9 and 1A1 genes. Pharmacogenet Genomics 15(5): 295-301.

Inoue T, Chung YS, Yashiro M, Nishimura S, Hasuma T, Otani S, Sowa M (1997) Transforming growth factor-beta and hepatocyte growth factor produced by gastric fibroblasts stimulate the invasiveness of scirrhous gastric cancer cells. Jpn J Cancer Res 88(2): 152-159.

Inoue $\mathrm{T}$, Kataoka $\mathrm{H}$, Goto K, Nagaike K, Igami K, Naka D, Kitamura N, Miyazawa K (2004) Activation of c-Met (hepatocyte growth factor receptor) in human gastric cancer tissue. Cancer Sci 95(10): 803-808.

Iwasaki H, Suda T (2009) Cancer stem cells and their niche. Cancer Sci 100(7): 1166-1172.

Jedema I, Barge RM, Frankel AE, Willemze R, Falkenburg JH (2004) Acute myeloid leukemia cells in G0 phase of the cell cycle that are unresponsive to conventional chemotherapy are sensitive to treatment with granulocytemacrophage colony-stimulating factor/diphtheria toxin fusion proteins. Exp Hematol 32(2): 188-194.

Kohne CH, Catane R, Klein B, Ducreux M, Thuss-Patience P, Niederle N, Gips M, Preusser P, Knuth A, Clemens M, Bugat R, Figer I, Shani A, Fages B, Di Betta D, Jacques C, Wilke HJ (2003) Irinotecan is active in chemonaive patients with metastatic gastric cancer: a phase II multicentric trial. Br J Cancer 89(6): 997-1001.

Kondo T, Setoguchi T, Taga T (2004) Persistence of a small subpopulation of cancer stem-like cells in the C6 glioma cell line. Proc Natl Acad Sci USA 101(3): 781-786.

Korzeniewska-Dyl I (2008) [Caspases-a new target in inflammation and cancer therapy?]. Pol Merkur Lekarski 24(139): 5-7.

Kuniyasu H, Yasui W, Kitadai Y, Yokozaki H, Ito H, Tahara E (1992) Frequent amplification of the c-met gene in scirrhous type stomach cancer. Biochem Biophys Res Commun 189(1): 227-232.

Li C, Wu JJ, Hynes M, Dosch J, Sarkar B, Welling TH, Pasca di Magliano M, Simeone DM (2011a) c-Met is a marker of pancreatic cancer stem cells and therapeutic target. Gastroenterology 141(6): 2218-2227 e5.
Li L, Bhatia R (2011) Stem cell quiescence. Clin Cancer Res 17(15): 4936-4941.

Li Y, Li A, Glas M, Lal B, Ying M, Sang Y, Xia S, Trageser D, GuerreroCazares H, Eberhart CG, Quinones-Hinojosa A, Scheffler B, Laterra J (2011b) c-Met signaling induces a reprogramming network and supports the glioblastoma stem-like phenotype. Proc Natl Acad Sci USA 108(24): 9951-9956.

Marth C, Daxenbichler G, Dapunt O (1986) Synergistic antiproliferative effect of human recombinant interferons and retinoic acid in cultured breast cancer cells. J Natl Cancer Inst 77(6): 1197-1202.

Nishii T, Yashiro M, Shinto O, Sawada T, Ohira M, Hirakawa K (2009) Cancer stem cell-like SP cells have a high adhesion ability to the peritoneum in gastric carcinoma. Cancer Sci 100(8): 1397-1402.

Pfaffl MW (2001) A new mathematical model for relative quantification in real-time RT-PCR. Nucleic Acids Res 29(9): e45.

Pommier Y, Pourquier P, Fan Y, Strumberg D (1998) Mechanism of action of eukaryotic DNA topoisomerase I and drugs targeted to the enzyme. Biochim Biophys Acta 1400(1-3): 83-105.

Reddiconto G, Toto C, Palama I, De Leo S, de Luca E, De Matteis S, Dini L, Passerini CG, Di Renzo N, Maffia M, Coluccia AM (2012) Targeting of GSK3beta promotes imatinib-mediated apoptosis in quiescent CD34+ chronic myeloid leukemia progenitors, preserving normal stem cells. Blood 119(10): 2335-2345.

Sattler M, Pride YB, Ma P, Gramlich JL, Chu SC, Quinnan LA, Shirazian S, Liang C, Podar K, Christensen JG, Salgia R (2003) A novel small molecule met inhibitor induces apoptosis in cells transformed by the oncogenic TPR-MET tyrosine kinase. Cancer Res 63(17): 5462-5469.

Shah AN, Summy JM, Zhang J, Park SI, Parikh NU, Gallick GE (2007) Development and characterization of gemcitabine-resistant pancreatic tumor cells. Ann Surg Oncol 14(12): 3629-3637.

Sunayama J, Matsuda K, Sato A, Tachibana K, Suzuki K, Narita Y, Shibui S, Sakurada K, Kayama T, Tomiyama A, Kitanaka C (2010) Crosstalk between the PI3K/mTOR and MEK/ERK pathways involved in the maintenance of self-renewal and tumorigenicity of glioblastoma stem-like cells. Stem Cells 28(11): 1930-1939.

Tahara E (2004) Genetic pathways of two types of gastric cancer. IARC Sci Publ 157: 327-349.

Toiyama Y, Yasuda H, Saigusa S, Matushita K, Fujikawa H, Tanaka K, Mohri Y, Inoue Y, Goel A, Kusunoki M (2012) Co-expression of hepatocyte growth factor and c-Met predicts peritoneal dissemination established by autocrine hepatocyte growth factor/c-Met signaling in gastric cancer. Int J Cancer 130(12): 2912-2921.

Wang JY, Hsieh JS, Chen CC, Tzou WS, Cheng TL, Chen FM, Huang TJ, Huang YS, Huang SY, Yang T, Lin SR (2004) Alterations of APC, c-met, and $\mathrm{p} 53$ genes in tumor tissue and serum of patients with gastric cancers. J Surg Res 120(2): 242-248.

Yashiro M, Chung YS, Inoue T, Nishimura S, Matsuoka T, Fujihara T, Sowa M (1996a) Hepatocyte growth factor (HGF) produced by peritoneal fibroblasts may affect mesothelial cell morphology and promote peritoneal dissemination. Int J Cancer 67(2): 289-293.

Yashiro M, Chung YS, Nishimura S, Inoue T, Sowa M (1995) Establishment of two new scirrhous gastric cancer cell lines: analysis of factors associated with disseminated metastasis. Br J Cancer 72(5): 1200-1210.

Yashiro M, Chung YS, Nishimura S, Inoue T, Sowa M (1996b) Peritoneal metastatic model for human scirrhous gastric carcinoma in nude mice. Clin Exp Metastasis 14(1): 43-54.

Yashiro M, Chung YS, Sowa M (1994) Role of orthotopic fibroblasts in the development of scirrhous gastric carcinoma. Jpn J Cancer Res 85(9): 883-886.

Yashiro M, Hirakawa K (2010) Cancer-stromal interactions in scirrhous gastric carcinoma. Cancer Microenviron 3(1): 127-135.

Yokozaki H, Yasui W, Tahara E (2001) Genetic and epigenetic changes in stomach cancer. Int Rev Cytol 204: 49-95.

Yonemura Y, Kaji M, Hirono Y, Fushida S, Tsugawa K, Fujimura T, Miyazaki I, Harada S, Yamamoto H (1996) Correlation between overexpression of c-met gene and the progression of gastric cancer. Int J Oncol 8(3): 555-560.

Zhao J, Zhang X, Xin Y (2011) Up-regulated expression of Ezrin and c-Met proteins are related to the metastasis and prognosis of gastric carcinomas. Histol Histopathol 26(9): 1111-1120.

This work is published under the standard license to publish agreement. After 12 months the work will become freely available and the license terms will switch to a Creative Commons AttributionNonCommercial-Share Alike 3.0 Unported License. 